\title{
The observation of the phenophase in six blueberry varieties
}

\author{
Tingyu Gou ${ }^{1,{ }^{*}}$, Can Luo ${ }^{1}$, Jiaqi Le ${ }^{1}$, Jun Wang ${ }^{1,{ }^{*},}$ Xun Wang ${ }^{2, a}$ \\ ${ }^{1}$ College of Horticulture, Sichuan Agricultural University, Chengdu, Sichuan, China \\ ${ }^{2}$ Institute of Pomology \& Olericulture, Sichuan Agricultural University, Chengdu, Sichuan, China \\ a wangxun0104@hotmail.com \\ *The first author
}

Keywords: Six blueberry varieties, phenophase, leaf, bloom, fruit.

Abstract. Blueberry was one of the five healthiest fruits in the world. We observed the phenophase in six blueberry varieties from the leaf buds germination period to the fruit maturity period. The six blueberry varieties included 'EM', 'Biloxi', 'Pink Lemonide', 'O'Neal', 'Sweetheart' and 'Brigitta', in Nanbao Mountain, Qionglai, Sicuan, China. We observed the growth period of leaves, deciduous period, blooming stages, blooming period and fruit period. The results showed that the different spans and changes of different varieties. The observation of the phenophase in six blueberry varieties was expected to provide data base for the blueberry's field management and agriculture production.

\section{Introduction}

Blueberry, belonging to Ericaceae family, Vaccinium genus, was a kind of perennial and evergreen or deciduous shrub. Blueberry fruit looks blue, as a kind of berry, also named "blue berry". Blueberry was round, small sized between $0.5 \mathrm{~g}$ and $5 \mathrm{~g}$, covered with white fruit powder, having a suitable acid and sweet flavor as well as nice smell. Importantly, blueberry was beneficial to our health with rich nutrition such as mineral, vitamin $\mathrm{C}$ and fiber's elements, especially the anthocyanin which protect people's microvessels in eyes by boosting our circulation. Besides, Rimand ${ }^{[1]}$ found some chemicals for cancer prevention such as picea tannin alcohol. As was mentioned above, blueberry has helpful health cwere function so that we can eat it to strengthen our immunity and prevent cancer. Blueberry, named as "golden berry", was recommended as one of the five healthiest fruits by FAO, for being famous for the rich and diverse health cwere composition in the world ${ }^{[2]}$.

The phenophase was natural season phenomena influenced by environmental factors, including climate, water and soil, climate being the dominant factor, in the period of the year ${ }^{[3]}$. The phenophase was important for agricultural production and field management. The observation of the phenophase can help us know the growth of blueberry, and then we can manage it better. For instance, when watering or fertilizing, preventing insect pests, we need to know which period was the best time to do it.

\section{Materials and methods}

Materials. Six varieties, including 'EM', 'Biloxi', 'Pink Lemonide', 'O'Neal', 'Sweetheart', and 'Brigitta', were used. Thirty trees for each variety were chosen. The blueberry plants were in orchard in Nanbao Mountain, Qionglai, Sicuan, China.

Methods. We observed the leaf buds germination period, leaf opening period, leaf-expansion period, deciduous period, flower bud sprouting period, floret separation period, initial bloom period, full bloom period, terminal bloom period, young fruit period, expanding period, coloring period and maturity period of the six blueberry varieties from middle February 2017 to the end of May 2017.

\section{Results}

The growth period of leaves. The growth period of leaves contains leaf buds germination, leaf opening period and leaf-expansion. Leaf buds germination period means that the leaf buds start to 
appear till it starts to open. The leaf opening period means that the leaf buds were opening from the curl state. The leaf-expansion period means that the leaf was growing bigger after opening completely.

We observed the leaf buds germination period, leaf opening period and leaf-expansion period these three periods. The observation of the growth period of leaves of the six varieties 'EM', 'Biloxi', 'Pink Lemonide', 'O'Neal', 'Sweetheart' and 'Brigitta' was showing in the Fig.1. From the Fig. 1, it showed that the whole growth period of leaves of 'EM' was the earliest in the six varieties. Its leaf buds germination was from $2 / 22$ to $2 / 28$, leaf opening period was from $2 / 28$ to $3 / 5$, leaf-expansion period was from $3 / 5$ to $3 / 11$. The growth period of leaves of Brigitta was the latest one that its leaf buds germination period was from $3 / 25$ to $4 / 1$, leaf opening period was from $4 / 1$ to $4 / 4$, leaf-expansion period was from $4 / 4$ to $4 / 8$. The growth period of leaves of 'Biloxi', 'Pink Lemonide' and 'Sweetheart' was similar that their leaf buds germination period was from $3 / 11$ to $3 / 18$, leaf opening period was from $3 / 18$ to $3 / 25$, leaf-expansion period was from $3 / 25$ to $4 / 1$. The growth period of leaves of O'Neal was earlier than the three varieties mentioned above that its leaf buds germination period was from $3 / 5$ to $3 / 11$, leaf opening period was from $3 / 11$ to $3 / 18$, leaf-expansion period was from $3 / 18$ to $3 / 25$.

The deciduous period. The deciduous period means that the leaves start to defoliate till all the leaves defoliate completely. The blueberry varieties of 'O'Neal', 'Brigitta' and 'Sweetheart' were evergreen shrubs while the varieties of 'EM', 'Biloxi' and 'Pink Lemonide' were deciduous shrubs. The observation of the deciduous period was showing in the Fig.2. From Fig.2, the blueberry tree of 'Biloxi' had a longer span in deciduous period covering twenty-nine days from $3 / 25$ to $4 / 22$. While the 'EM' and 'Pink Lemonide' both were covering twenty-three days from 4/1 to 4/22.

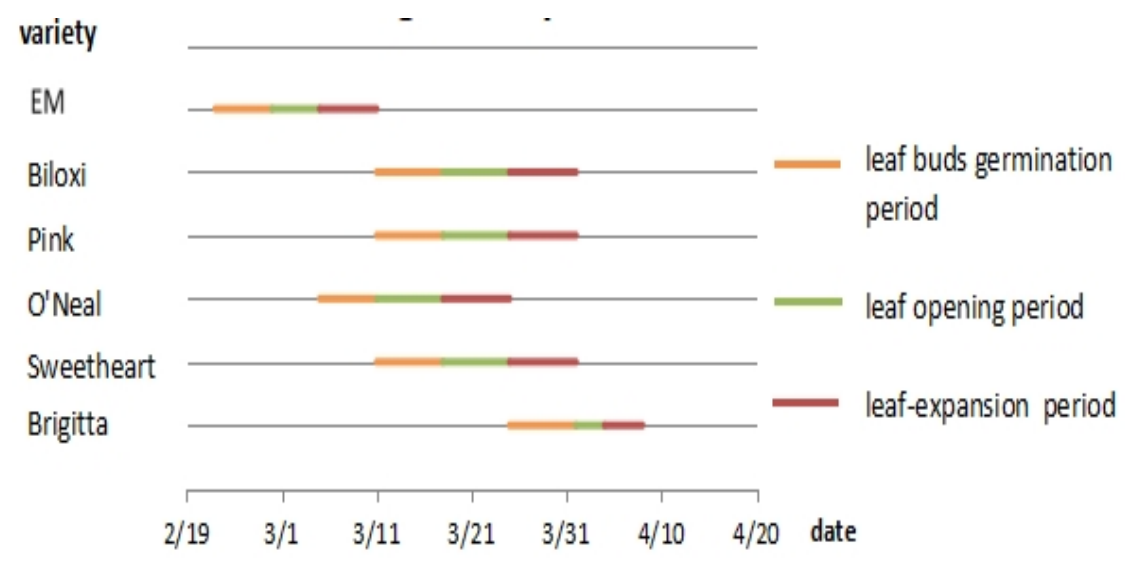

Fig. 1 The growth period of leaves

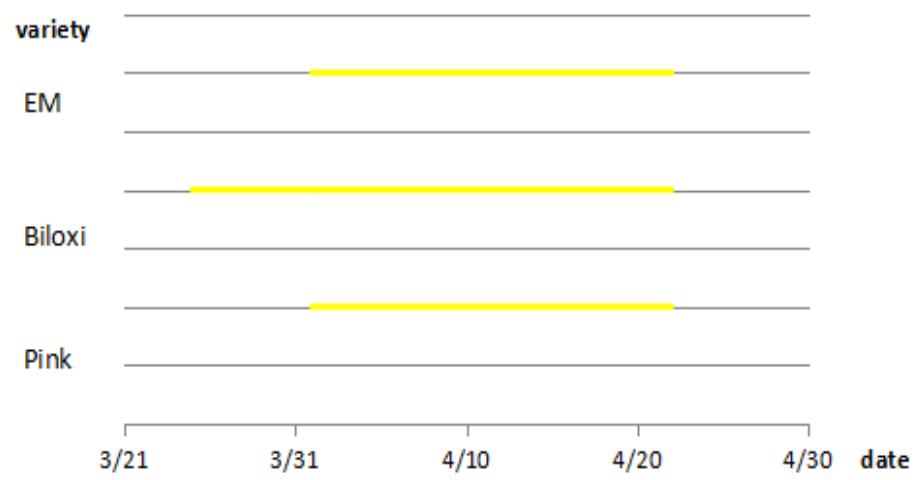

Figure 2 The deciduous period

The early stage blooming development. The early stage blooming development here contained flower bud sprouting period and floret separation period. The flower bud sprouting period means that 
the flower bud starts to appear till the raceme starts to separate. The floret separation period is during the period that the raceme is separating. The observation of the early stage blooming development of the sex varieties was showing in the Fig.3. The early stage blooming development of 'Brigitta' was the latest one in the six blueberry varieties that flower bud sprouting period was from $3 / 25$ to $3 / 28$ and floret separation period was from 3/28 to 4/1. 'EM' and 'O'Neal' were both the earliest in the six blueberry varieties that the flower bud sprouting period was from $2 / 28$ to $3 / 5$ while floret separation period was from $3 / 5$ to $3 / 11$. 'Biloxi' and 'Sweetheart' take the second place that flower bud sprouting period was from $3 / 5$ to $3 / 11$ while floret separation period was from 3/11 to 3/18. Meanwhile, 'Pink Lemonide"s flower bud sprouting period was from $3 / 11$ to $3 / 18$ and floret separation period was from $3 / 18$ to $3 / 25$.

The blooming period. The blooming period here contains initial bloom period, full bloom period and terminal bloom period. Initial bloom period means that the blossom quantities are at twenty-five percent. Full bloom period means that the blossom quantities are at the percentage between twenty-five percent and seventy-five percent. Terminal bloom period means that the blossom quantities are at the percentage between seventy-five percent and ninety-five percent[ ]. The observation of the blooming period was showing in the fig.4. From the Fig.4, 'Sweetheart'"s blooming period was the longest in the six varieties from $3 / 18$ to $4 / 15$, in detail, initial bloom period was from $3 / 18$ to $3 / 25$, full bloom period was from $3 / 25$ to $4 / 4$, terminal bloom period was from $4 / 4$ to 4/15. Also we can see that 'EM', 'Biloxi' and 'O'Neal' were earlier and 'Pink Lemonide', 'Brigitta' and 'Sweetheart' were later.

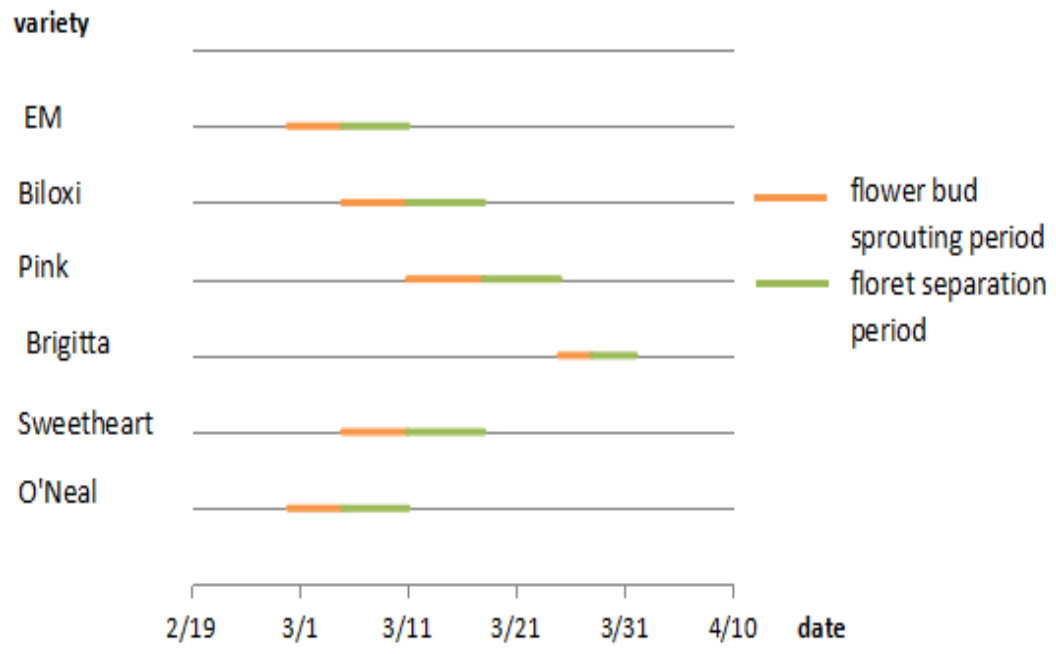

Figure 3 The early stage blooming development

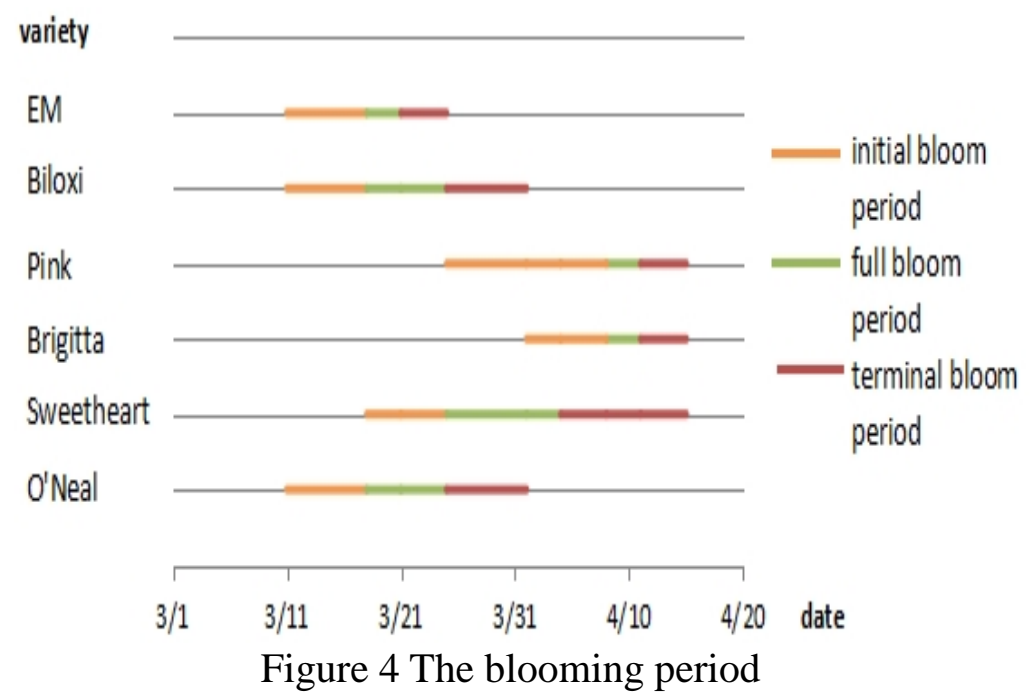


The fruit period. The fruit period contains young fruit period, expanding period, coloring period and maturity period. Young fruit period is the prophase of the fruit growth. Expanding period is the period that the fruit was expanding. Coloring period means that the blueberry's color is turning red and blue from green. Maturity period means that the blueberry becoming mature. The observation of the fruit period was showing in the Fig.5, From the figure, the fruit period of the 'Sweetheart', 'Brigitta' and 'Pink Lemonide' differs little that the start maintains between 4/8 and 4/15, while the end maintains between 6/4 and 6/11. The fruit period of 'EM' was the earliest in the six varieties that was from $3 / 25$ to $5 / 20$. The maturity period of 'EM' was the earliest in the six varieties so that it can obtain better economic benefits than other five varieties for other five varieties were still immature, when it was hot in the marketing.

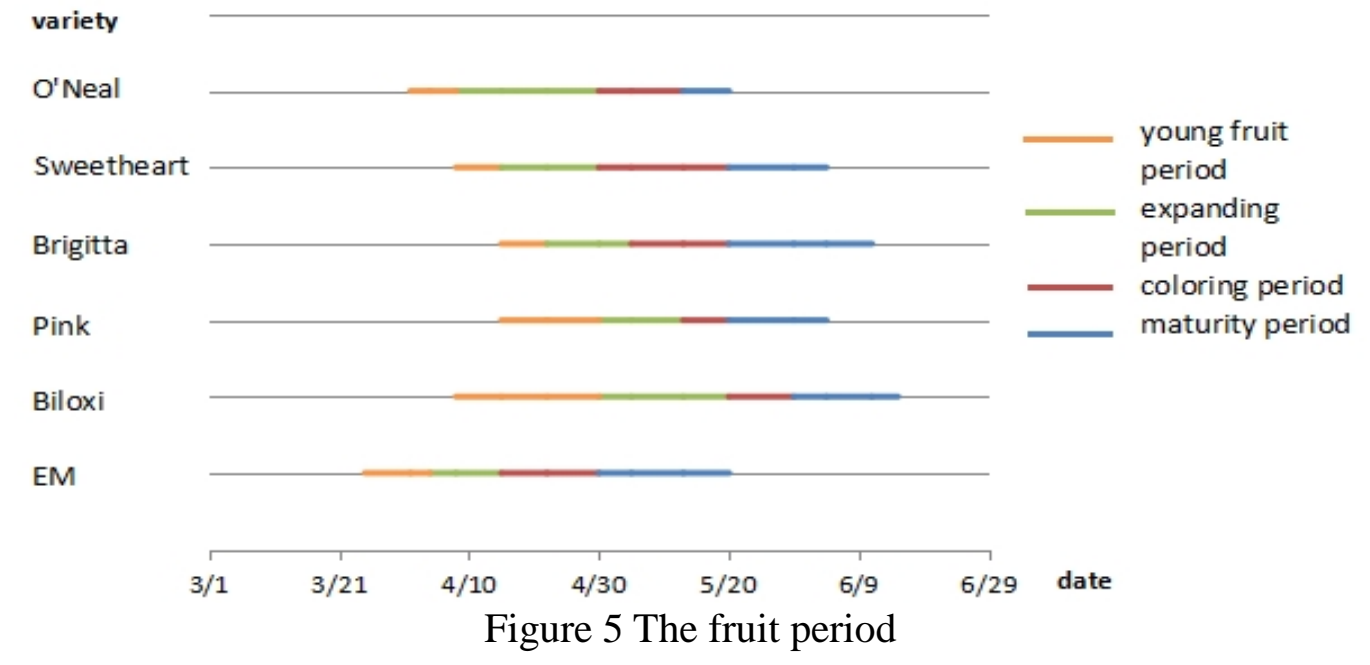

\section{Conclusions}

The observation of the six blueberry varieties was beneficial to our field management and agriculture production. For example, colonization was before the leaf buds germination period. In blooming period and fruit expanding period blueberry trees need more water to boost the growth of fruits but control the water in the maturity period. Besides, when fertilizing and preventing insect pests, we need to know the various periods of blueberry trees. For example, prevent the botrytis cinerea in the initial bloom period, prevent producing dry fruit before the blooming period, and prevent scarabs in the blooming period[ ]. We know the blueberry's maturity period so that we can pick blueberries in exact date after preparing for it completely. Nevertheless, the phenophase influenced by the climate and the climate being different every year, the observation of the six blueberry varieties will have a little change later. Anyway, the observation of the blueberry trees also will be essential in the field management and agriculture production for the items above.

\section{References}

[1] A.M. Rimando, W. Kalt, J.B. Magee, J. Dewey and J.R. Ballington: The Journal of Agricultural and Food Chemwastry Vol. 52(2004), p. 4713-4719

[2] L. Cheng, H. Deng, S.M. Ren and W. Guo: Agricultural Science and Technology Newsletter Vol. 4(2010), p. 20-22

[3] M.J. Wang, J. Sun, Y.D. Duan, S. Hou, W.W. Liang and H.J. Du: Northern Horticulture Vol. 20(2016), p. 52-54

[4] X.S. Wang, W.H. Ma, D.B. Xu, T.T. Yuan, Y.Q. Liu and L. Wu: Journal of Northeast Agricultural Sciences Vol. 41(2012), p. 100-103

[5] Y.Zhen: Jilin Agriculture Vol. 6(2018), p. 85 